\title{
OPEN A novel RNA-mediated mechanism causing down-regulation of insulating promoter interactions in human embryonic stem cells
}

\author{
Yingjuan Liu ${ }^{1}$, Simon G. Williams ${ }^{1}$, Hayden R. Jones ${ }^{1}$, Bernard D. Keavney ${ }^{1,2}$ \&
} Mun-Kit Choy ${ }^{1 凶}$

The genome-wide promoter interactome is primarily maintained and regulated by architectural proteins such as CTCF and cohesin. However, some studies suggest a role for non-coding RNAs (ncRNAs) in this process. We aimed to characterise the regulatory role of RNA-mediated promoter interactions in the control of gene expression. We integrated genome-wide datasets of RNAchromatin and promoter-genome interactions in human embryonic stem cells (hESCs) to identify putative RNA-mediated promoter interactions. We discovered that CTCF sites were enriched in RNAPIRs (promoter interacting regions co-localising with RNA-chromatin interaction sites) and genes interacting with RNA-PIRs containing CTCF sites showed higher expression levels. One of the long noncoding RNAs (IncRNAs) expressed in hESCs, Syntaxin 18-Antisense 1 (STX18-AS1), appeared to be involved in an insulating promoter interaction with the neighbouring gene, MSX1. By knocking down STX18-AS1, the MSX1 promoter-PIR interaction was intensified and the target gene (MSX1) expression was down-regulated. Conversely, reduced $M S X 1$ promoter-PIR interactions, resulting from CRISPR-Cas9 deletion of the PIR, increased the expression of MSX1. We conclude that STX18-AS1 RNA antagonised local CTCF-mediated insulating promoter interactions to augment gene expression. Such down-regulation of the insulating promoter interactions by this novel mechanism may explain the higher expression of genes interacting with RNA-PIRs linked to CTCF sites.

Gene regulation involves the formation of three dimensional (3D) chromosomal interactions to bring distal genomic regulatory elements to spatial proximity of gene promoters ${ }^{1,2}$. The occurrence of these long-range chromosomal interactions is not only mediated by protein factors that are key players in maintaining genome architecture such as CCCTC binding factor (CTCF), Yin Yang 1 (YY1) and the Mediator and cohesin complexes, but also the widespread non-coding RNAs (ncRNAs) transcribed in the genome ${ }^{3}$. For example, Thymocyte Differentiation factor (ThymoD) ncRNA activates cohesin-dependent looping to juxtapose the enhancer and promoter of $B$ cell lymphoma/leukaemia $11 b(B c l 11 b)$ gene into a single loop domain for T cell fate specifying expression ${ }^{4}$. Functional intergenic repeating RNA element (Firre), a long intergenic noncoding RNA (lincRNA) localises across a $5 \mathrm{Mb}$ domain around its transcription site and this domain interacts with several trans-chromosomal loci ${ }^{5}$. Deletion of the Firre locus leads to the loss of the trans-chromosomal interactions, suggesting a role for Firre lincRNA in 3D chromosomal organisation ${ }^{5}$.

Although the mechanism by which ncRNAs facilitate long-range chromosomal interactions is largely unknown, it has been hypothesised that this function is made possible by their flexible modular domains that bind proteins, DNAs and other RNAs and bring these regulatory components to close proximity ${ }^{6}$. Architectural proteins such as the Mediator complex, CTCF and YY1 have been shown to associate with RNAs ${ }^{7-10}$. Depletion of Mediator's subunits or activating ncRNAs reduces the chromatin looping between the activating ncRNAs and their target genes ${ }^{7}$, whilst mutations at CTCF's RNA interaction sites cause the loss of chromatin loops ${ }^{10}$. In the case of cohesin, the positioning of the complex requires active transcription in the mouse genome ${ }^{11}$ and displacement of it depends on the 'read-through' transcription (extending past the 3 ' ends of genes) in the host

\footnotetext{
${ }^{1}$ Division of Cardiovascular Sciences, The University of Manchester, Manchester M13 9PT, UK. ${ }^{2}$ Manchester Heart Institute, Manchester University NHS Foundation Trust, Manchester M13 9WL, UK. ${ }^{\circledR}$ email: munkit.choy@ manchester.ac.uk
} 
A

Overlapped with

RNA-DNA interaction regions?
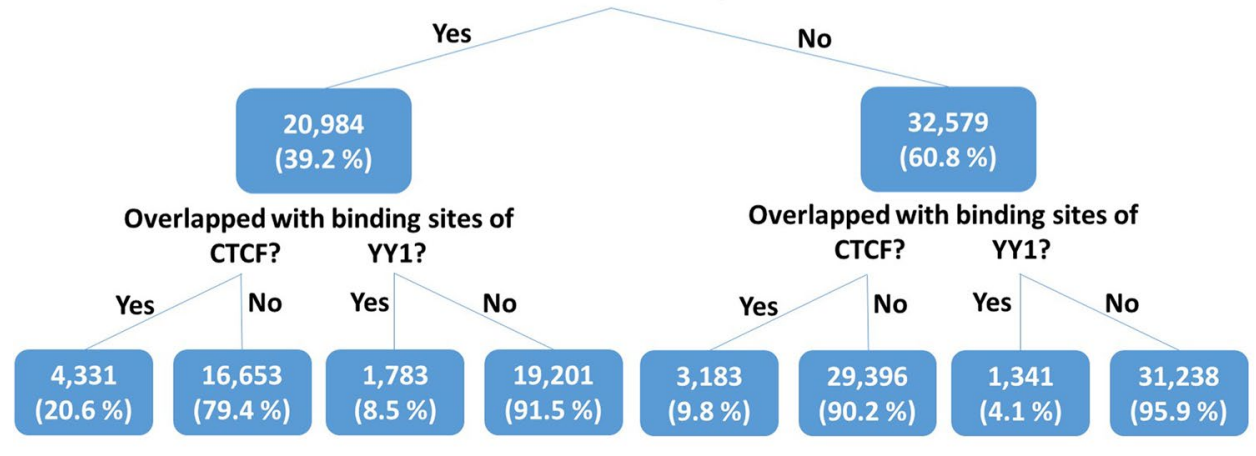

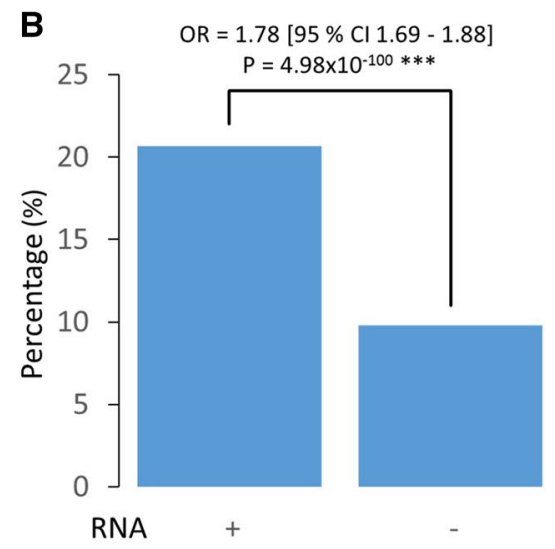

CTCF

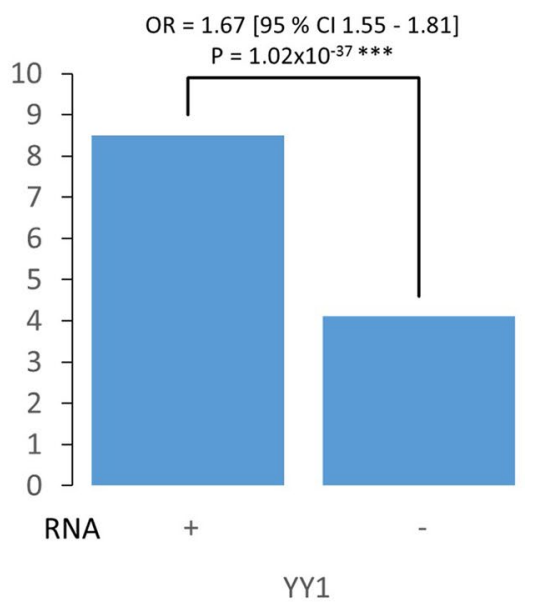

Figure 1. Enrichment of CTCF or YY1 binding sites in promoter interacting regions interacting with RNAs (RNA-PIRs). (A) Numbers and percentages of PIRs in human embryonic stem cells (hESCs) interacting with RNAs and containing binding sites of CTCF or YY1. (B) Odds Ratios (ORs) and p-values of CTCF or YY1 site enrichment were estimated from binary logistic regression analyses including PIR size as a covariate. ${ }^{* *}$ p-values obtained from binary logistic regression analyses were lower than the p-values obtained from the same analyses performed with 1000 randomised CTCF or YY1 site sets (permutation $\mathrm{P}<1.0 \times 10^{-3}$ ).

cell during the influenza A infection to decompact the locus ${ }^{12}$. This mechanism is an example of how the transcription itself, but not the resulting RNAs, can affect 3D genome organisation ${ }^{3}$.

In this study, we aimed to characterise the regulatory role of RNA-mediated promoter interactions in the control of gene expression. Recently, genome-wide and targeted methods have been devised to map RNA-DNA contacts in the genome, providing the experimental capability to understand the role of RNAs in chromosomal interactions $\mathrm{s}^{13-17}$. Promoter-genome and RNA-genome mapping have been performed on human embryonic stem cells (hESCs) and their differentiated cells to understand the maintenance of pluripotency and the differentiation process ${ }^{15,18,19}$. Here we integrate promoter capture $\mathrm{HiC}(\mathrm{PCHiC})$ and mapping RNA-genome interactions (MARGI) datasets of H9 hESCs to identify and investigate putative RNA-mediated promoter interactions ${ }^{15,19}$, and elucidate the role of a functionally important long noncoding RNA (lncRNA), Syntaxin 18-Antisense 1 (STX18-AS1), in one of the promoter interactions of hESCs. SNPs in the region of the STX18-AS1 gene have been associated with atrial septal defects of the heart in multiple studies ${ }^{20-23}$.

\section{Results}

CTCF and YY1 binding sites are enriched in RNA-PIRs. We found that of the 53,563 unique promoter interaction regions (PIRs) in the genome of H9 hESCs previously reported ${ }^{19}, 20,984$ of them overlapped $^{2}$ with genomic regions that are interacting with RNAs as identified through proximity MARGI (pxMARGI) ${ }^{15}$ (Fig. 1A). We hypothesised that RNAs play a role in the promoter-genome interactions, therefore we first investigated whether PIRs interacting with RNAs (RNA-PIRs) have enrichments for architectural protein binding such as CTCF and YY1. While CTCF is responsible for large chromosomal interactions to provide a 3D architecture for genes and their regulatory elements, more specific promoter-enhancer interactions are mediated by YY $1^{24}$. 


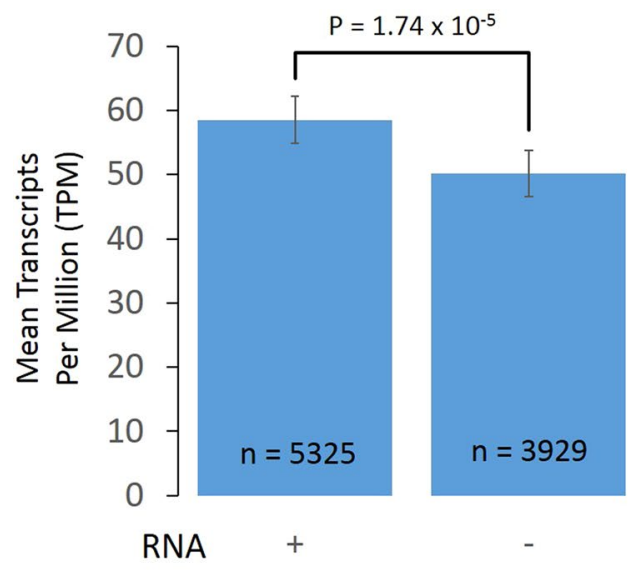

CTCF

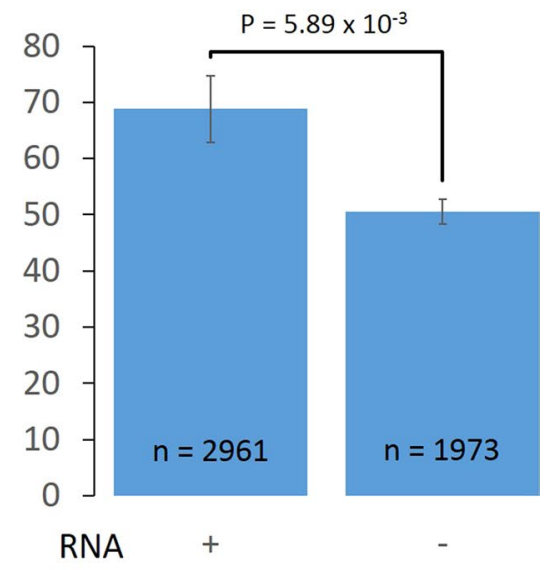

YY1

Figure 2. Average expression levels (mean Transcript Per Million [TPM]) of genes interacting with two categories of promoter interacting regions (PIRs): RNA-PIRs with CTCF or YY1 binding sites, and nonRNAPIRs with CTCF or YY1 binding sites. Pairwise p-values were obtained from Mann-Whitney tests.

We obtained CTCF and YY1 binding sites of H1 hESCs from the Encyclopaedia of DNA Elements (ENCODE) ${ }^{25,26}$ and intersected them with the PIRs (Supplementary Table 1). $20.6 \%$ of RNA-PIRs contained CTCF binding sites, whereas only $9.8 \%$ of PIRs that are not interacting with RNAs (nonRNA-PIRs) had CTCF binding sites (odds ratio $[\mathrm{OR}]=1.78 ; 95 \% \mathrm{CI}=1.69-1.88 ; \mathrm{P}=4.98 \times 10^{-100}$ ). Similarly, $8.5 \%$ of RNA-PIRs contained YY1 binding sites, while $4.1 \%$ of nonRNA-PIRs contained these sites $(\mathrm{OR}=1.67 ; 95 \% \mathrm{CI}=1.55-1.81$; $\mathrm{P}=1.02 \times 10^{-37}$ ) (Fig. 1). The PIR size, which correlates with the probability of overlap, was included as a covariate in the binary logistic regression (Fig. 1B). We generated null distributions for the p-values of the binary logistic regression analyses by subjecting 1000 randomly generated genomic interval sets matching those in the CTCF or YY1 binding site sets to the same analyses. None of the p-values produced using randomised sets was lower than the actual $p$-values, hence the $p$-values of the binary logistic regression analyses were significantly lower than their null distributions (permutation $\mathrm{P}<1.0 \times 10^{-3}$ ).

PIRs are HindIII fragments and not the biological demarcations for the chromatin interacting regions. Therefore the intersection of PIRs and CTCF sites only indicates their physical proximity for the convenience of bioinformatic investigations, but not the actual sites where specific CTCF-mediated promoter interactions occur.

Genes interacting with RNA-PIRs containing CTCF orYY1 binding sites have higher expression levels than those interacting with nonRNA-PIRs containing CTCF or YY1 binding sites. To understand if the expression of genes interacting with RNA-PIRs containing CTCF or YY1 sites are regulated differentially compared to those interacting with nonRNA-PIRs containing CTCF or YY1 sites, we obtained previously reported transcriptomic data of $\mathrm{H} 9 \mathrm{hESCs}^{19}$ and calculated average expression levels of genes interacting with the two categories of PIRs: (1) RNA-PIRs with CTCF or YY1 binding sites, or (2) nonRNA-PIRs with CTCF or YY1 binding sites (Fig. 2).

Between the categories, genes interacting with RNA-PIRs containing CTCF or YY1 sites appeared to have significantly higher expression levels $\left(\mathrm{P}=1.74 \times 10^{-5}\right.$ for CTCF sites and $\mathrm{P}=5.89 \times 10^{-3}$ for $\mathrm{YY} 1$ sites), while genes interacting with nonRNA-PIRs with CTCF or YY1 sites had lower expression levels (Fig. 2). From these results, RNAs seemed to have up-regulated genes in the presence of CTCF or YY1, through long-range promoter-genome interactions in the nucleus.

The co-regulation of RNAs and CTCF at PIRs plays an important role in biological processes in hESCs. Given that RNA-PIRs were enriched for more CTCF or YY1 sites and genes interacting with RNA-PIRs containing CTCF or YY1 sites exhibited higher expression levels, we hypothesised that the genes interacting with a PIR where both RNAs and CTCF/YY1 co-localised are the genes responsible for the biological functions of hESCs. We looked into the ontology of genes interacting with the RNA-PIRs containing CTCF or YY1 sites using g:Profiler ${ }^{27}$ (Supplementary Table 2). We compared the unique list of genes interacting with RNA-PIRs containing CTCF (3456 genes) or YY1 (2309 genes) sites with the unique list of genes interacting with nonRNA-PIRs containing CTCF (2060 genes) or YY1 (1321 genes) sites (Fig. 3).

$\mathrm{g}$ :Profiler returned the biological processes of genes that were significantly overrepresented in the unique gene lists (adjusted $\mathrm{P}<0.01$ ). The genes interacting with RNA-PIRs containing CTCF sites were significantly enriched in a wide range of development and cellular regulation processes (Supplementary Table 2). However, genes interacting with nonRNA-PIRs containing CTCF sites showed much fewer significant biological processes and no overlap when compared to genes interacting with RNA-PIRs containing CTCF sites, as only an "animal organ development" process was returned (Fig. 3). This suggests that the genes interacting with RNA-PIRs 


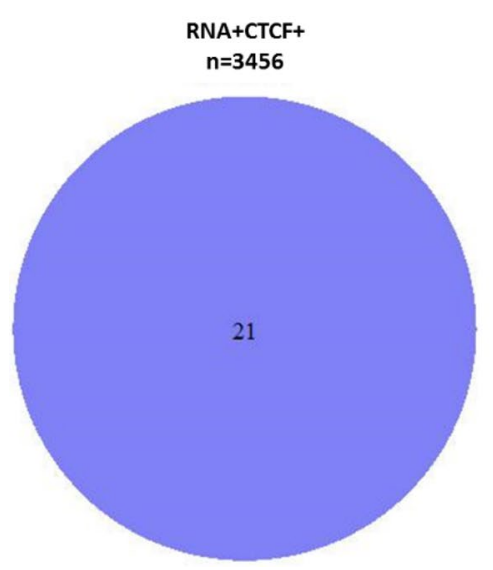

\section{Numbers of \\ Enriched Biological Processes \\ for Target Gene Lists}

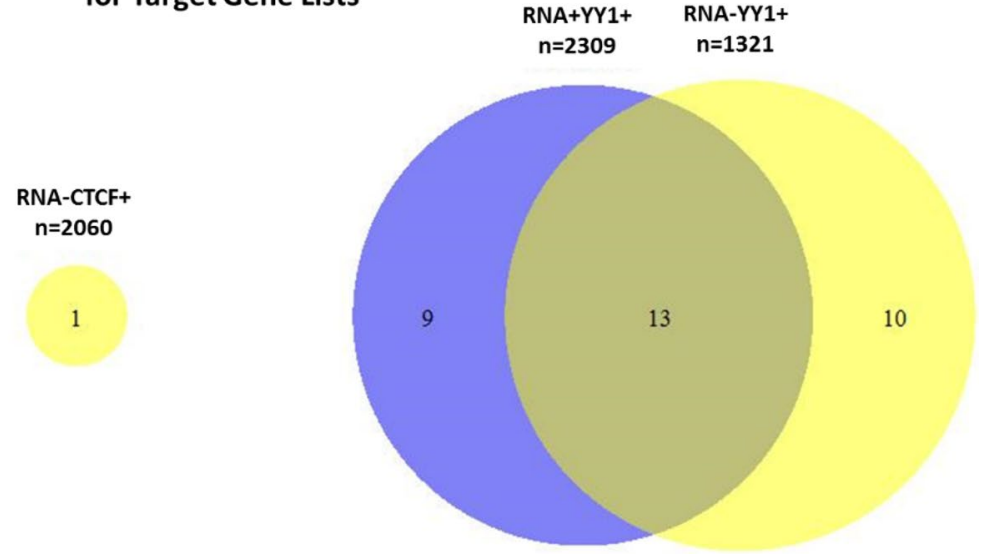

Figure 3. Numbers of biological processes (g:Profiler) that were significantly overrepresented (adjusted $\mathrm{p}<0.01$ ) in unique lists of genes interacting with RNA-PIRs or nonRNA-PIRs containing CTCF or YY1 sites.

were involved in a wider range of the biological functions happening in hESCs than the genes interacting with nonRNA-PIRs.

Conversely, genes interacting with RNA-PIRs containing YY1 sites and genes interacting with nonRNAPIRs containing YY1 sites belonged to similar biological processes. The two lists of genes shared 13 processes in development and cellular regulation (Fig. 3). Therefore, the results suggest that RNAs play a more important role in their co-regulation with CTCF than YY1. RNAs and CTCF, but not CTCF alone, regulate more genes involved in a broader range of biological functions in hESCs, whereas YY1, with or without RNAs, regulates genes belonging to a similar range of ontologies.

STX18-AS1 RNA represses an insulating promoter-PIR interaction of the neighbouring MSX1 gene. We further investigated the RNA-CTCF co-regulation in promoter interactions by experimentally manipulating a promoter interaction between the promoter of Msh Homeobox 1 (MSX1), a transcription factor gene that plays a role in vertebrate embryogenesi ${ }^{28}$, and an RNA-PIR that is interacting with Syntaxin 18-Antisense 1 (STX18-AS1), an lncRNA in hESCs. STX18-AS1 gene contains genetic variants associated with atrial septal defects of the heart in genome-wide association studies (GWAS ${ }^{20-23}$. As an RNA-PIR, We hypothesised that $M S X 1$ promoter-PIR interaction is insulating the expression of MSX1 due to the association of RNA-RIRs with CTCF/YY1 sites as seen in Fig. 1B. In Fig. 4A, it is apparent that hESC CTCF sites are abundant between the PIR of MSX1 and the promoter of MSX1, but YY1 sites are very sparse in the region, suggesting that CTCF is the main factor that mediates this chromatin interaction. We also hypothesised that STX18-AS1 RNA upregulates MSX1 expression, as the RNA presence in CTCF-mediated promoter interactions led to higher expression levels of the target gene of promoter interactions as seen in Fig. 2.

Firstly, we knocked down the expression of STX18-AS1 RNA using paired guide RNA (gRNA) CRISPRCas9 technology $y^{29}$ to delete the first and second exons of STX18-AS1 gene (Fig. 4A,B). We then checked the effect of STX18-AS1 knock-down on the MSX1 gene by quantifying MSX1 gene expression using qPCR and MSX1 promoter-PIR interaction using 3C-qPCR. MSX1 expression was down-regulated but MSX1 promoterPIR interaction was up-regulated when STX18-AS1 RNA was knocked down (Fig. 4B). These findings support the hypotheses that the MSX1 promoter-PIR interaction acts as a CTCF-mediated interaction with an insulating effect on MSX1 expression. Our results would be consistent with STX18-AS1 RNA antagonising this interaction to upregulate the expression of MSX1. We confirmed this result by knocking down STX18-AS1 using Antisense Locked Nucleic Acid (LNA) GapmeR technology ${ }^{30}$ targeting the second exon of STX18-AS1 gene. MSX1 was similarly down-regulated as a consequence (Fig. 4C) - the confirmation with GapmeR demonstrating that no additional regulatory element in the region removed by CRISPR-Cas9 was influencing the result of the CRISPRCas9 experiment. Thus, STX18-AS1 RNA acted as a positive regulator for MSX1 expression regardless of the knock-down technology used.

In order to verify the insulating effect of MSX1 promoter-PIR interaction, we down-regulated the interaction by deleting part of the PIR that does not overlap with the STX18-AS1 gene using paired gRNA CRISPR-Cas9 (Fig. 4A,D). The PIR deletion did not affect the expression of STX18-AS1 RNA but reduced the amount of MSX1 promoter-PIR interaction (Fig. 4D). In this case, the expression of $M S X 1$ was up-regulated, providing additional evidence that the MSX1 promoter-PIR interaction is indeed a CTCF-mediated interaction with an insulating effect on MSX1 expression.

When designing the gRNA constructs for deleting STX18-AS1 gene and the PIR interacting with MSX1, regions rich in MSX1-targeting expression quantitative trait loci (eQTLs) were avoided (Fig. 4A). Therefore the experimental effects on MSX1 caused by the deletions were aptly explained by the manipulations of STX18AS1 expression and MSX1 promoter-PIR interaction, while no known cis-regulatory elements of MSX1 were 
A Genomic Features/ CRISPR Positions

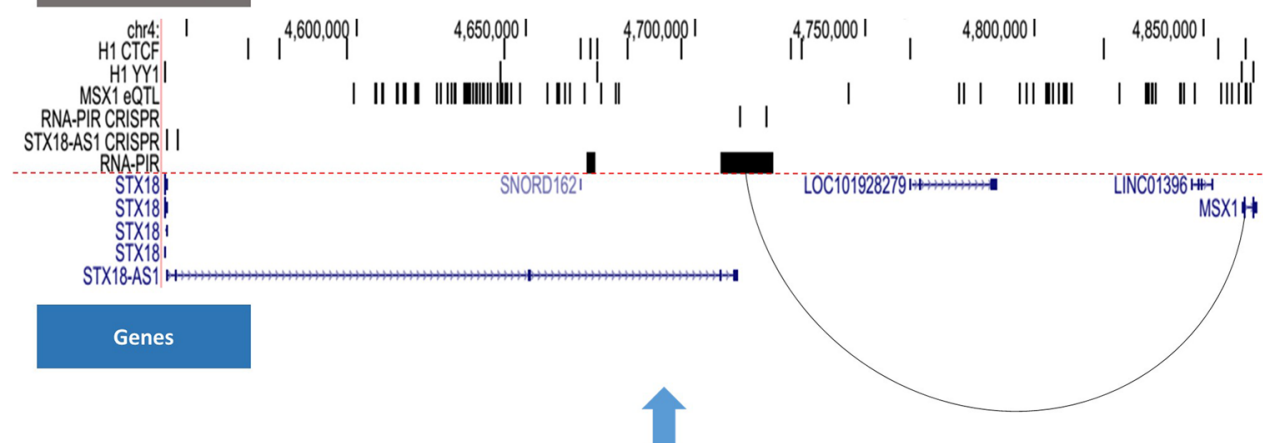

B

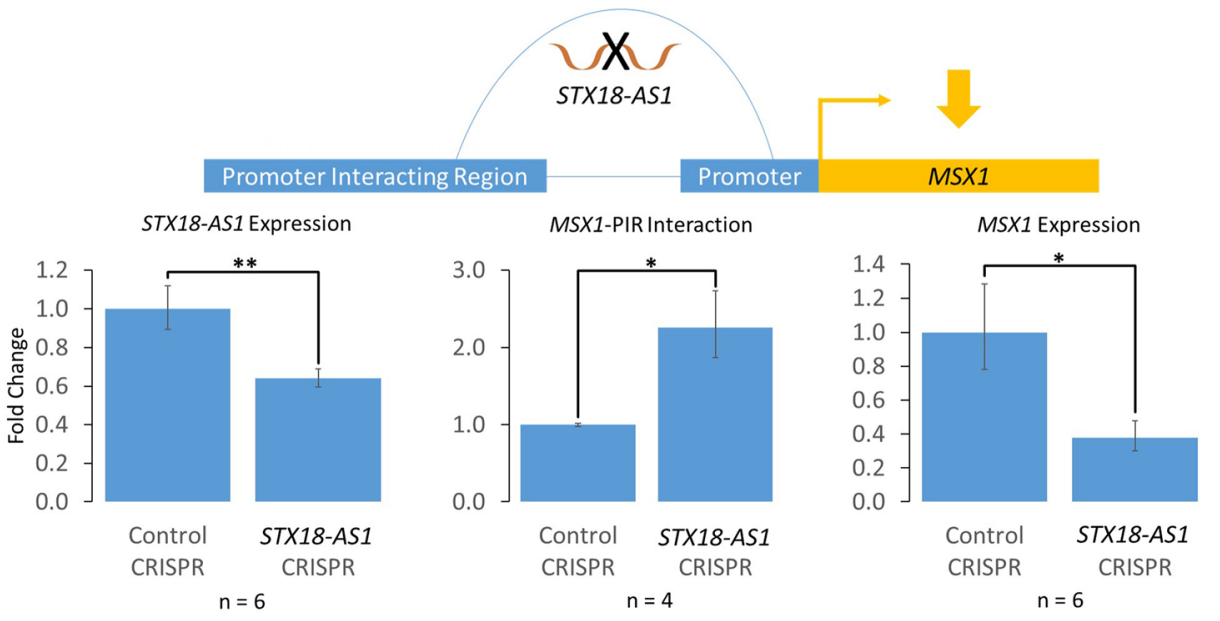

C
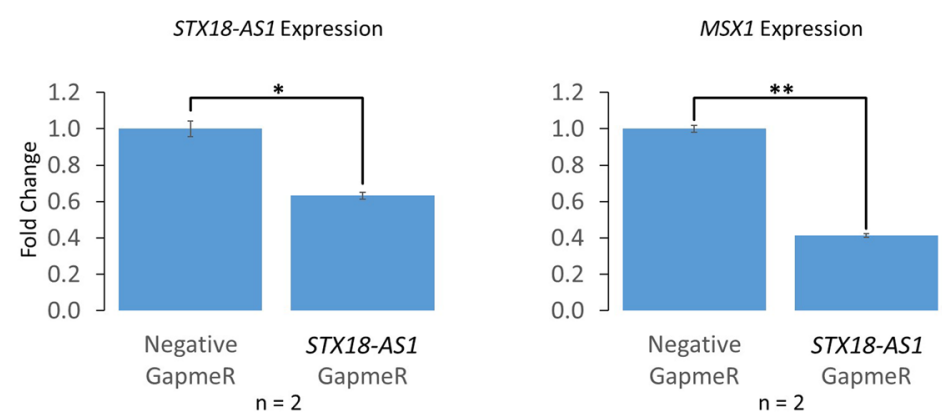

Figure 4. Chromosomal interaction between $M S X 1$ promoter and its promoter interacting region (PIR) mediated by a noncoding RNA, STX18-AS1. (A) Genomic locations of MSX1 and STX18-AS1 genes, CTCF and YY1 binding sites in H1 human embryonic stem cells (hESCs), GTEx expression quantitative trait loci (eQTLs) targeting MSX1 gene, guide RNA (gRNA) sequences for deleting the first two exons of STX18-AS1 gene and part of MSX1 PIR using CRISPR-Cas9, and PIRs associated with RNAs (the MSX1 promoter-PIR interaction was represented by an arc line). (B) Effects of STX18-AS1 down-regulation by deleting part of STX18-AS1 gene using CRISPR-Cas9 on MSX1 expression and MSX1 promoter-PIR interaction. (C) Effects of STX18-AS1 downregulation by a specific GapmeR on STX18-AS1 and MSX1 expression levels. (D) Effects of downregulated MSX1 promoter-PIR interaction by deleting part of MSX1 RNA-PIR using CRISPR-Cas9 on expression levels of STX18-AS1 and MSX1. E) Effects of STX18-AS1 and MSX1 RNA-PIR CRISPR-Cas9 deletions, and STX18-AS1 GapmeR on expression levels of STX18, SOX2 and OCT4. ${ }^{\star} \mathrm{P}<0.05 ;{ }^{*} \mathrm{P}<0.01$. All $\mathrm{p}$-values were obtained from T tests with Bonferroni correction if multiple comparisons apply.

disrupted. All the CRISPR-Cas9 and GapmeR manipulations in this study did not significantly affect the expression of the upstream STX18 gene or pluripotent marker genes SOX2 and OCT4 (Fig. 4E). 
D

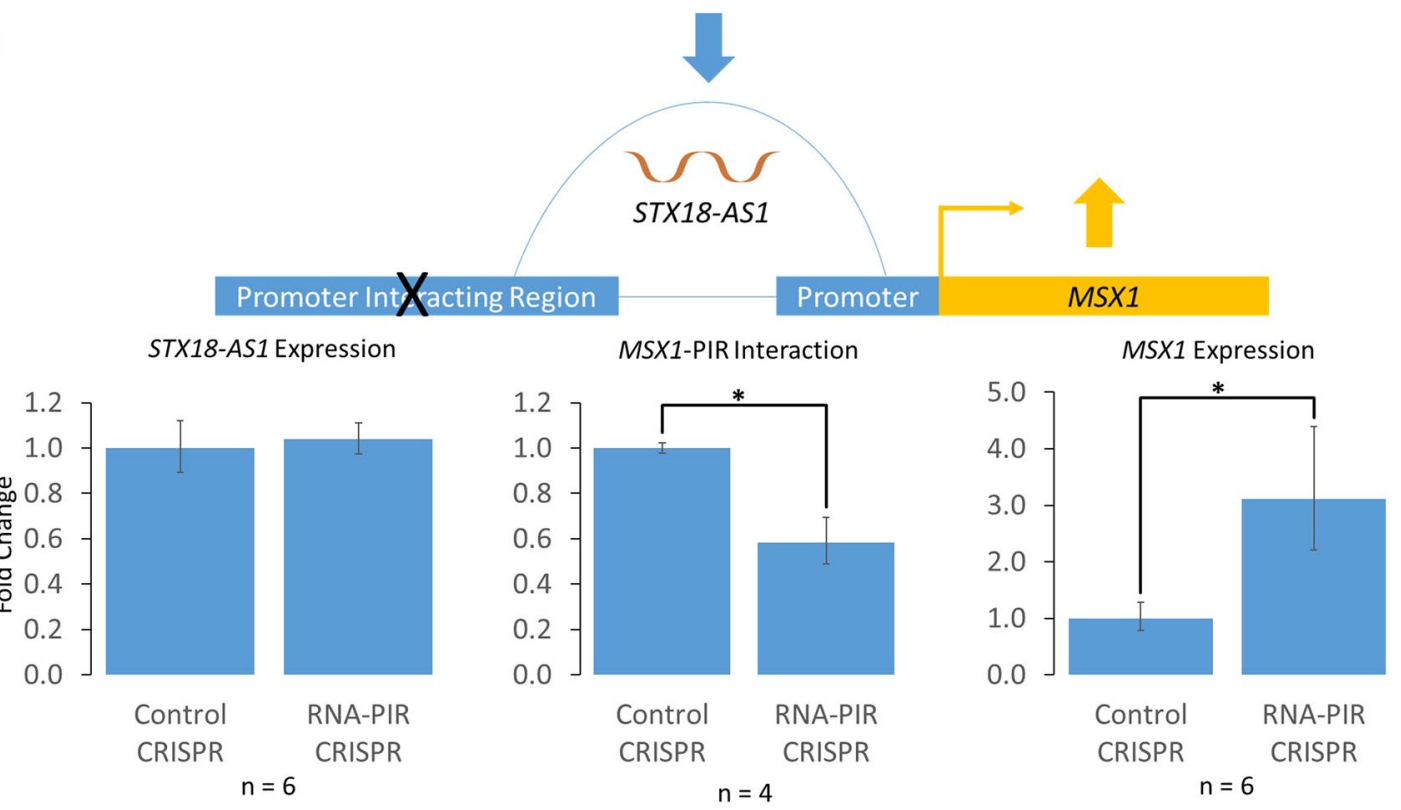

E

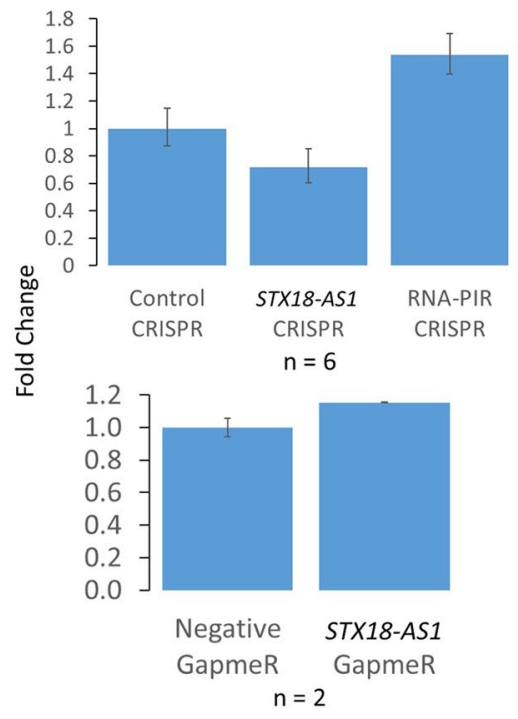

SOX2 Expression

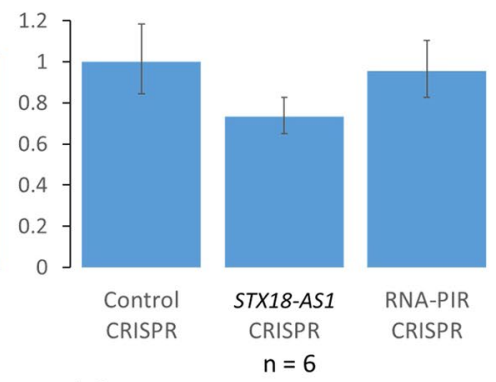

$\left.\begin{array}{l}1.2 \\ 1.0 \\ 0.8 \\ 0.6 \\ 0.4 \\ 0.2 \\ 0.0\end{array}\right]$

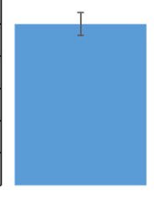

Negative GapmeR
OCT4 Expression
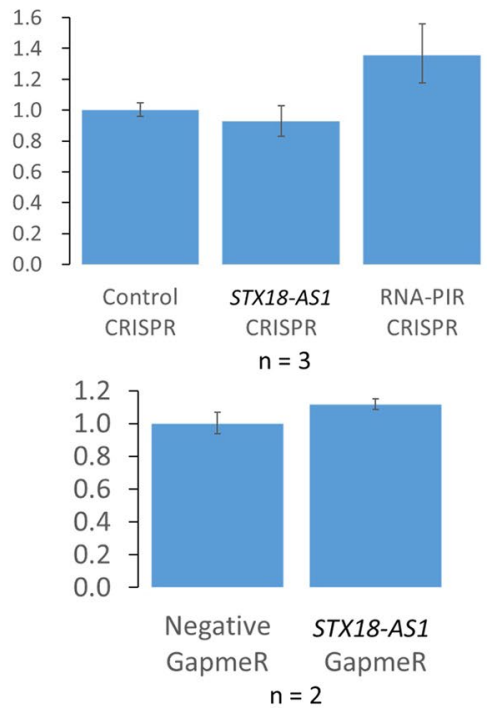

Figure 4. (continued)

\section{Discussion}

In this study we identified putative genome-wide RNA-mediated promoter-genome interactions in hESCs by integrating PCHiC and MARGI datasets and dissecting the role of an exemplar RNA in such an interaction. We provide evidence that RNAs co-regulate promoter interactions with CTCF. Firstly, binding sites of CTCF were more enriched in RNA-PIRs than nonRNA-PIRs. Secondly, genes interacting with RNA-PIRs containing CTCF sites had higher expression levels in the cells. Thirdly, genes interacting with RNA-PIRs containing CTCF sites, but not nonRNA-PIRs with CTCF sites, were overrepresented in a broad range of biological processes such as development and cellular regulation. On the other hand, genes interacting with PIRs containing YY1 sites belonged to a similar range of biological processes, with or without RNAs. Taking all the findings together, they suggest that RNAs and CTCF work together in maintaining and regulating promoter interactions.

By knocking down the RNA (STX18-AS1) of one of the putative RNA-mediated promoter-genome interactions (MSX1 promoter-PIR interaction), the promoter interaction was intensified and the expression of the target gene $(M S X 1)$ was down-regulated. This suggests that the promoter interaction is repressive or insulating. The majority of promoter-genome interactions are maintained by architectural proteins such as CTCF and cohesin; indeed most promoter-genome interactions are lost when CTCF and cohesin are degraded ${ }^{31}$. Given the abundance of CTCF sites in the genomic region and CTCF being the major insulating factor to mediate promoter interactions, they suggest that the RNA (STX18-AS1) represses a CTCF-mediated insulating interaction with the promoter of the target gene (MSX1). The expression level of MSX1 was up-regulated by knocking down the 
MSX1 promoter-PIR interaction through deleting part of the PIR, indicating that the promoter interaction is both necessary and sufficient to regulate MSX1 expression. Given the high expression levels shown by the genes interacting with RNA-PIRs containing CTCF sites, it supports the conclusion that the CTCF-mediated insulating promoter interactions can be suppressed by RNAs, just like the MSX1 promoter-PIR interaction that insulates the MSX1 expression is being suppressed by STX18-AS1 RNA in hESCs.

\section{Materials and methods}

hESC maintenance. H9 (WA09) hESC line was a publicly available cell line obtained from WiCell, cultured in monolayers and maintained according to WiCell Feeder-Independent Pluripotent Stem Cell Protocols (SOPSH-002) using mTeSR1 medium (STEMCELL Technologies).

CRISPR-Cas9 and lentiviral transduction. gRNA sequences (provided in Supplementary Table 3) used to delete STX18-AS1 gene and MSX1 PIR in H9 hESC were cloned into pLentiCRISPRv2 (Addgene; plasmid 52961) according to published paired gRNA design ${ }^{29}$ using human U6 promoter for both gRNA sequences. The cloned pLentiCRISPRv2 and second-generation packaging plasmids psPAX2 (Addgene; plasmid 12260) and pMD2.G (Addgene; plasmid 12259) were co-transfected into HEK293T cells (cultured in DMEM (Gibco) supplemented with $10 \%$ FBS and $1 \times$ penicillin-streptomycin at $37{ }^{\circ} \mathrm{C}$ and $5 \% \mathrm{CO}_{2}$ ) in the proportion of 4:3:2 using Lipofectamine 2000 (Invitrogen) for lentivirus production. Lentivirus-containing media were collected $48 \mathrm{~h}$ after transfection, centrifuged to remove cell debris and $0.45 \mu \mathrm{m}$ filtered before transducing H9 hESC monolayers ( $40-60 \%$ confluency) in the presence of 33\% v/v mTESR1 media (STEMCELL Technologies), $8 \mu \mathrm{g} / \mathrm{ml}$ polybrene (Millipore) and $10 \mu \mathrm{M}$ ROCK inhibitor. $24 \mathrm{~h}$ post-transduction, second transduction was repeated with fresh lentivirus-containing media. $24 \mathrm{~h}$ after the second transduction, the transduced $\mathrm{H} 9 \mathrm{hESC}$ were selected in $0.8 \mu \mathrm{g} / \mathrm{ml}$ puromycin media for $48 \mathrm{~h}$. The strength of puromycin was less than the standard for better hESC survival, therefore the 48 -h puromycin selection step was repeated every time the cells were passaged. The deletions of STX18-AS1 gene and MSX1 PIR were confirmed by PCR and Sanger sequencing (primers and product sizes are provided in Supplementary Table 3; results are in Supplementary Figs. 1 and 2). All CRISPR-Cas9 experiments were controlled by cells transduced with an empty pLentiCRISPRv2 plasmid.

Antisense LNA GapmeR experiment. The GapmeR targeting STX18-AS1/LOC100507266 lncRNA (NR_037888.1) was designed using EXIQON LNA GapmeR On-Line Design Tool (http://www.exiqon.com/ gapmers). Both STX18-AS1 and negative GapmeRs (details provided in Supplementary Table 3) were supplied by Qiagen/Exiqon at HPLC purification grade. GapmeRs were transfected to cells using Lipofectamine RNAiMAX according to the manufacture's instruction (Invitrogen). Briefly for each 12-well of cells at $\sim 60 \%$ confluency ( $1 \mathrm{ml}$ of medium), $10 \mu$ Lipofectamine RNAiMAX and 50 pmol GapmeR were added to cells and incubated at $37^{\circ} \mathrm{C}$ for $24 \mathrm{~h}$. The same procedure was repeated after $24 \mathrm{~h}$ and cells were incubated for another $24 \mathrm{~h}$ before the collection.

$3 \mathrm{C}$ library preparation. 3C libraries were prepared according to a published protocol ${ }^{32}$. In summary, 20 million hESCs were fixed by $2 \%$ formaldehyde (Agar Scientific; R1026) for $10 \mathrm{~min}$ at room temperature, quenched by cold $0.125 \mathrm{M}$ glycine ( $5 \mathrm{~min}$ at room temperature followed by $15 \mathrm{~min}$ on ice), flash-frozen in liquid nitrogen and stored at $-80^{\circ} \mathrm{C}$. The cross-linked cells were lysed and the chromatin digested with HindIII at $37^{\circ} \mathrm{C}$ for overnight. Then in-nucleus ligation was carried out at $16^{\circ} \mathrm{C}$ for $4-6 \mathrm{~h}$ before reverse-crosslinking was achieved by overnight treatment of proteinase $\mathrm{K}$ at $65^{\circ} \mathrm{C}$. The DNA was purified by RNaseA treatment for $60 \mathrm{~min}$ at $37^{\circ} \mathrm{C}$, sequential phenol and phenol-chloroform extractions, and ethanol-precipitation at $-20^{\circ} \mathrm{C}$ for overnight. On the following day, two further phenol-chloroform extractions and a second overnight precipitation were performed to further purify the DNA. The promoter interaction junctions between two HindIII fragments were confirmed by PCR and Sanger sequencing (primers and band sizes are provided in Supplementary Table 3; results for MSX1 and ERCC Excision Repair 3 (ERCC3) interactions (positive/internal control) are in Supplementary Figs. 3 and 4).

Polymerase chain reaction (PCR). Genomic DNA (gDNA) was extracted from cells using PureLink Genomic DNA Mini Kit (Invitrogen) and total RNA using Trizol (Invitrogen). First-strand complementary DNA (cDNA) was synthesised from DNase (Promega) treated total RNA using M-MLV Reverse Transcriptase (Promega) and both random hexamer and oligo(dT) primers (Promega). PCR was performed using OneTaq $2 \times$ Master Mix (New England BioLabs) and the primers provided in Supplementary Table 3. For quantitative PCR (qPCR), gene expression was quantified by TaqMan assays (Applied Biosystems; assay details are provided in Supplementary Table 3) using a ViiA7 qPCR system (Applied Biosystems). For each qPCR reaction, $\sim 40 \mathrm{ng}$ cDNA templates were used with TaqMan Gene Expression Master Mix (Applied Biosystems; stock concentration $2 \times$ ) and the TaqMan assays (Applied Biosystems; stock concentration 20x). Promoter interactions in 3C libraries were quantified using SYBR Green PCR Master Mix (Applied Biosystems) and primers (information provided in Supplementary Table 3) in a ViiA7 qPCR system (Applied Biosystems). Three replicates were done for each qPCR reaction with the programme of $95^{\circ} \mathrm{C}$ for $10 \mathrm{~min}, 40 \times\left(95^{\circ} \mathrm{C}\right.$ for $15 \mathrm{~s}, 60^{\circ} \mathrm{C}$ for $\left.1 \mathrm{~min}\right)$. Importin 8 (IPO8) was included as the internal control for expression quantification, while ERCC3 interaction was used as the positive/internal/positive control for interaction quantification ${ }^{33}$.

Data processing and statistical analyses. RNA-chromatin interactions were obtained from http://syste msbio.ucsd.edu/margi/ (pxMARGI) ${ }^{15}$. To compare to the promoter-PIR interactions, coordinates in hg38 were 
converted to hg19 using UCSC liftover function (https://genome.ucsc.edu/). RNA or DNA regions with ambiguous mapping between genome builds or that mapped to 'unlocalized' or 'unplaced' regions were removed from further analyses. This resulted in 734,970 unique RNA-DNA interactions and 601,055 unique RNA-interacting DNA regions. Unique PIRs from $\mathrm{H} 9 \mathrm{hESCs}^{19}$ excluding interchromosomal interacting regions $(53,563)$ were then overlapped with these RNA-interacting DNA regions. A total of 20,984 of these PIRs were found to overlap RNA-interacting DNA regions, whereas the remaining 32,579 PIRs had no evidence of RNA interaction from these data.

Promoter-PIR interactions with evidence of accompanying RNA interaction, and those without RNA interaction, were assessed for overlap with CTCF and YY1 sites using the ENCODE CTCF and YY1 sites from H1 hESC cells (Consortium, 2012; Consortium et al., 2020). CTCF or YY1 sites overlapping PIRs (RNA or non-RNA) were counted using a consensus of all 5 ENCODE CTCF and 2 ENCODE YY1 sets (downloadable from https:// genome.ucsc.edu/; “Uniform TFBS" and "ENCODE 3 TFBS” tracks). For greater confidence in the overlaps, PIR and CTCF/YY1 overlaps were only counted if all datasets agreed (i.e. all 5 in the case of CTCF overlap and 2 in the case of YY1 overlap). Any degree of intersection between the PIRs and these high-confidence feature sets was included. We performed binary logistic regression, using PIR length as a covariate, to determine statistical significance of the difference between PIRs with/without RNA and CTCF/YY1 intersection. For negative controls, we generated consensus genomic intervals from the 5 CTCF and 2 YY1 datasets respectively. These were randomly shuffled within the genome using Bedtools (v2.27.1; https://bedtools.readthedocs.io/en/latest/). 1000 randomly generated interval sets were then assessed for their intersection with PIRs through the same statistical analysis as the actual data.

Gene promoters interacting with PIRs either associated with RNAs, CTCF or YY1 sites were assessed for the expression levels of their respective genes in H9 hESCs. H9 hESC RNA-seq dataset was downloaded from GEO (gene expression omnibus) accession GSE86821 (Freire-Pritchett et al., 2017). Raw data from the two replicates were pooled and transcript expression values determined using Kallisto (Bray et al., 2016) and the GRCh38 rel79 transcriptome from Ensembl (https://www.ensembl.org/). The mean expression values in terms of Transcripts Per Million (TPM) for genes identified in each overlapping PIR set ("RNA and CTCF", "CTCF-only", "RNA-only" and "neither") were calculated and compared.

g:Profiler (https://biit.cs.ut.ee/gprofiler/gost) ${ }^{27}$ was used to identify the overrepresentations of biological processes in gene lists. eQTLs were obtained from GTEx V8 (https://gtexportal.org/home/).

Data from qPCR were analysed using the $2^{\wedge}(-\Delta \Delta \mathrm{Ct})$ method as previously described ${ }^{34}$. According to this method, means and standard errors are calculated from $\Delta \mathrm{CT}$ values.

Standard statistical methods mentioned were performed with relevant $\mathrm{R}$ packages or other publicly available tools. Sample scripts and steps of this study are available on https://github.com/keavneylab/PIR-RNA-CTCF.

Received: 10 February 2021; Accepted: 15 November 2021

Published online: 01 December 2021

\section{References}

1. Mifsud, B. et al. Mapping long-range promoter contacts in human cells with high-resolution capture Hi-C. Nat. Genet. 47, 598-606 (2015).

2. Hughes, J. R. et al. Analysis of hundreds of cis-regulatory landscapes at high resolution in a single, high-throughput experiment. Nat. Genet. 46, 205-212 (2014).

3. Schoenfelder, S. \& Fraser, P. Long-range enhancer-promoter contacts in gene expression control. Nat. Rev. Genet. 20, 437-455 (2019).

4. Isoda, T. et al. Non-coding transcription instructs chromatin folding and compartmentalization to dictate enhancer-promoter communication and $\mathrm{T}$ cell fate. Cell 171, 103-119 (2017).

5. Hacisuleyman, E. et al. Topological organization of multichromosomal regions by the long intergenic noncoding RNA Firre. Nat. Struct. Mol. Biol. 21, 198-206 (2014).

6. Guttman, M. \& Rinn, J. L. Modular regulatory principles of large non-coding RNAs. Nature 482, 339-346 (2012).

7. Lai, F. et al. Activating RNAs associate with Mediator to enhance chromatin architecture and transcription. Nature 494, 497-501 (2013).

8. Kung, J. T. et al. Locus-specific targeting to the X chromosome revealed by the RNA interactome of CTCF. Mol. Cell 57, 361-375 (2015).

9. Sigova, A. A. et al. Transcription factor trapping by RNA in gene regulatory elements. Science 350, 978-981 (2015).

10. Saldana-Meyer, R. et al. RNA interactions are essential for CTCF-mediated genome organization. Mol. Cell 76, 412-4225 (2019).

11. Busslinger, G. A. et al. Cohesin is positioned in mammalian genomes by transcription, CTCF and Wapl. Nature 544, 503-507 (2017).

12. Heinz, S. et al. Transcription elongation can affect genome 3D structure. Cell 174, 1522-1536 (2018).

13. Bell, J. C. et al. Chromatin-associated RNA sequencing (ChAR-seq) maps genome-wide RNA-to-DNA contacts. Elife 7, e27024 (2018).

14. Li, X. et al. GRID-seq reveals the global RNA-chromatin interactome. Nat. Biotechnol. 35, 940-950 (2017).

15. Sridhar, B. et al. Systematic mapping of RNA-chromatin interactions in vivo. Curr. Biol. 27, 610-612 (2017).

16. Quinodoz, S. A. et al. Higher-order inter-chromosomal hubs shape 3D genome organization in the nucleus. Cell 174, 744-757 (2018).

17. Mumbach, M. R. et al. HiChIRP reveals RNA-associated chromosome conformation. Nat. Methods 16, 489-492 (2019).

18. Choy, M. K. et al. Promoter interactome of human embryonic stem cell-derived cardiomyocytes connects GWAS regions to cardiac gene networks. Nat. Commun. 9, 2526 (2018).

19. Freire-Pritchett, P. et al. Global reorganisation of cis-regulatory units upon lineage commitment of human embryonic stem cells. Elife 6, e21926 (2017).

20. Cordell, H. J. et al. Genome-wide association study of multiple congenital heart disease phenotypes identifies a susceptibility locus for atrial septal defect at chromosome 4p16. Nat. Genet. 45, 822-824 (2013). 
21. Pei, K. et al. Association between the $4 \mathrm{p} 16$ susceptibility locus and the risk of atrial septal defect in population from Southeast China. Pediatr. Cardiol. 37, 120-124 (2016).

22. Zhao, B. et al. Replication of the $4 \mathrm{p} 16$ susceptibility locus in congenital heart disease in Han Chinese populations. PLoS ONE 9, 107411 (2014).

23. Zhao, L. et al. Association between the European GWAS-identified susceptibility locus at chromosome $4 \mathrm{p} 16$ and the risk of atrial septal defect: A case-control study in Southwest China and a meta-analysis. PLoS ONE 10, e0123959 (2015).

24. Weintraub, A. S. et al. YY1 is a structural regulator of enhancer-promoter loops. Cell 171, 1573-1588 (2017).

25. Consortium E. P. An integrated encyclopedia of DNA elements in the human genome. Nature 489, 57-74 (2012).

26. Consortium E. P. et al. Perspectives on ENCODE. Nature 583, 693-698 (2020).

27. Reimand, J. et al. g:Profiler-A web-based toolset for functional profiling of gene lists from large-scale experiments. Nucleic Acids Res. 35, W193-200 (2007).

28. Liang, J. et al. MSX1 mutations and associated disease phenotypes: Genotype-phenotype relations. Eur. J. Hum. Genet. 24, 16631670 (2016)

29. Vidigal, J. A. \& Ventura, A. Rapid and efficient one-step generation of paired gRNA CRISPR-Cas9 libraries. Nat. Commun. 6, 8083 (2015).

30. Crooke, S. T. et al. Antisense technology: An overview and prospectus. Nat. Rev. Drug Discov. 20, 427-453 (2021).

31. Thiecke, M. J. et al. Cohesin-dependent and -independent mechanisms mediate chromosomal contacts between promoters and enhancers. Cell Rep. 32, 107929 (2020).

32. Naumova, N. et al. Analysis of long-range chromatin interactions using Chromosome Conformation Capture. Methods 58, 192-203 (2012).

33. Kubiak, M. et al. Chromosome Conformation Capture reveals two elements that interact with the PTBP3 (ROD1) transcription start site. Int. J. Mol. Sci. 20, 242 (2019).

34. Livak, K. J. \& Schmittgen, T. D. Analysis of relative gene expression data using real-time quantitative PCR and the 2(-Delta Delta C(T)) method. Methods 25, 402-408 (2001).

\section{Acknowledgements}

We would like to thank the members of Keavney Lab (Division of Cardiovascular Sciences, The University of Manchester) for their constructive comments. This work was supported by the following British Heart Foundation (BHF) grants: $\mathrm{CH} / 13 / 2 / 30154$ and RG/15/12/31616. H.R.J was supported by BHF 4-Year PhD Studentship Programme FS/19/60/34899 and The University of Manchester.

\section{Author contributions}

Conceptualisation, M.-K.C.; funding acquisition, B.D.K.; supervision, M.-K.C. and B.D.K.; wet laboratory investigation, Y.L.; bioinformatic investigation, M.-K.C., S.G.W. and H.R.J.; writing-original draft, M.-K.C.; writingreview and editing, all authors.

\section{Competing interests}

The authors declare no competing interests.

\section{Additional information}

Supplementary Information The online version contains supplementary material available at https://doi.org/ 10.1038/s41598-021-02373-1.

Correspondence and requests for materials should be addressed to M.-K.C.

Reprints and permissions information is available at www.nature.com/reprints.

Publisher's note Springer Nature remains neutral with regard to jurisdictional claims in published maps and institutional affiliations.

(c) (i) Open Access This article is licensed under a Creative Commons Attribution 4.0 International License, which permits use, sharing, adaptation, distribution and reproduction in any medium or format, as long as you give appropriate credit to the original author(s) and the source, provide a link to the Creative Commons licence, and indicate if changes were made. The images or other third party material in this article are included in the article's Creative Commons licence, unless indicated otherwise in a credit line to the material. If material is not included in the article's Creative Commons licence and your intended use is not permitted by statutory regulation or exceeds the permitted use, you will need to obtain permission directly from the copyright holder. To view a copy of this licence, visit http://creativecommons.org/licenses/by/4.0/.

(c) The Author(s) 2021 